\title{
Oxidative Stress Delays Prometaphase/Metaphase of the First Cleavage in Mouse Zygotes via the MAD2L1-Mediated Spindle Assembly Checkpoint
}

\author{
Que Wu, Zhiling Li, Yue Huang, Diting Qian, Man Chen, Wanfen Xiao, and Bin Wang \\ Reproductive Center, The First Affiliated Hospital of Shantou University Medical College, Shantou University, Shantou, \\ Guangdong, China
}

Correspondence should be addressed to Zhiling Li; stlizhiling@126.com

Received 19 May 2017; Revised 30 July 2017; Accepted 14 August 2017; Published 25 September 2017

Academic Editor: Serafina Perrone

Copyright ( 2017 Que Wu et al. This is an open access article distributed under the Creative Commons Attribution License, which permits unrestricted use, distribution, and reproduction in any medium, provided the original work is properly cited.

\begin{abstract}
In zygotes, DNA damage delays the first cleavage to enable repair. Our previous study found that 0.03 mM hydrogen peroxide $\left(\mathrm{H}_{2} \mathrm{O}_{2}\right)$ was the minimum concentration required for induction of oxidative DNA damage in mouse zygotes and that this represented the most similar situation to the clinical phenomenon. In this study, we quantified the cleavage rates of cells in blastocysts at different developmental stages, followed by immunofluorescence to detect activation of $\gamma$-H2A histone family member X (a marker of DNA damage) in zygotes to confirm that oxidative DNA damage was induced in $\mathrm{H}_{2} \mathrm{O}_{2}$-treated zygotes. Monitoring H3S10P (phosphorylation of Ser10 on histone H3; a prometaphase/metaphase marker) levels at different hour postinsemination revealed that treatment of zygotes with $0.03 \mathrm{mM} \mathrm{H}_{2} \mathrm{O}_{2}$ resulted in a prometaphase/metaphase delay. Furthermore, immunofluorescence staining for mitotic arrest deficient 2-like 1 and the protein kinase TTK, components of the spindle assembly checkpoint (SAC), suggested that this delay possibly involved SAC activation. These studies of the relationships between oxidative stress and SAC can promote the success rate of in vitro fertilization.
\end{abstract}

\section{Introduction}

Although many infertile couples have birthed children with the help of assisted reproduction technologies, these methods continue to suffer from excessive developmental failure due to differences between in vitro culture conditions and the in vivo environment, including energy sources, growth factors, $\mathrm{pH}$, and atmosphere. Suboptimal embryo-culture conditions in vitro can induce high levels of reactive oxygen species (ROS) in embryos and alter embryo development [1-3]. Excessive ROS generation can affect cellular function, signal transduction, and gene expression, as well as lipid peroxidation and physical DNA damage, resulting in nuclear and mitochondrial DNA-strand breaks in embryos $[1,4,5]$. High levels of ROS represent a major contributing factor in vitro to developmental blockage in zygotes [6]. However, due to ethical limitations, research cannot be performed on human embryos; therefore, in our studies, we treat mouse zygotes with hydrogen peroxide $\left(\mathrm{H}_{2} \mathrm{O}_{2}\right)$ to simulate the clinical phenomenon.

In the clinic, embryonic aneuploidy in gametes or early embryos represents the leading cause of in vitro fertilization (IVF) failure [7]. This occurs when abnormal chromosomes or their abnormal segregation events are present in early stage embryos [8], resulting in excessive developmental failure in embryos [9]. However, some embryos can appear normal by day 3, although many do not reach the blastocyst stage $[10,11]$. This phenomenon might be related to ROS [12]; therefore, this study tested a hypothesis suggesting a possible relationship between ROS and aneuploidy occurrence in in vitro-cultured zygotes. The occurrence of aneuploidy is tightly connected with spindle assembly checkpoint (SAC) function, which ensures correct segregation of chromosomes into daughter cells and prevents premature metaphase-anaphase transition until all chromosomes successfully attach to the bipolar spindle with proper 
tension [13]. The SAC can prevent aneuploidy by delaying chromosome segregation at prometaphase or metaphase in response to unattached kinetochores and DNA damage during preimplantation development $[9,14-16]$.

Because the SAC is involved in monitoring the metaphase-anaphase transition, to determine whether the cell cycle checkpoint mediates metaphase delay in response to oxidative stress, we analyzed the cell cycle kinetics of the first cleavage event in the zygote by monitoring the number of 2-cell blastocysts with both nuclei enriched for histone H3 phosphorylated on Ser10 (H3S10P), a marker of prometaphase/metaphase $[15,17]$. In mammalian cells, $\mathrm{H} 3 \mathrm{~S} 10 \mathrm{P}$ initially occurs within a subset of genes in late G2interphase cells but subsequently spreads along the chromosomes until completion at prometaphase, after which $\mathrm{H} 3 \mathrm{~S} 10 \mathrm{P}$ is still evident at the metaphase plate [17, 18]. Delay of H3S10P events in whole chromatin is indicative of prometaphase/metaphase delay and SAC activation [15].

To determine whether the SAC is engaged in response to oxidative stress damage in zygotes, we examined the localization of mitotic arrest deficient 2-like 1 (MAD2L1; also known as MAD2) and the protein kinase TTK by immunofluorescence staining. The end of prometaphase in mitosis correlates with checkpoint inactivation and disappearance of MAD2L1, a key player involved in SAC function [19]. MAD2L1 is observed in a punctate pattern throughout the cytoplasm but translocates to the nucleus following DNA damage and SAC activation [15, 20]. By contrast, the TTK dualspecificity protein kinase, which has been implicated in regulation of mitotic progression, is encoded by human and mouse monopolar spindle 1 (MPS1) [9] and is essential for the SAC. TTK can directly prevent anaphase onset in the presence of misaligned chromosomes. Additionally, TTK activity at the kinetochore stimulates MAD1-dependent conformational changes in MAD2L1 from an open to a closed form $[21,22]$, which is crucial for inhibition of anaphasepromoting complex/cyclosome (APC/C) [6, 23-26], the ubiquitin ligase that controls anaphase onset.

To characterize the effects of oxidative stress on zygotes, our previous studies used different doses of hydrogen peroxide $\left(\mathrm{H}_{2} \mathrm{O}_{2}\right)$ to treat mouse zygotes at $7 \mathrm{~h}$ postinsemination (hpi) to simulate the clinical phenomenon of oxidative damage in zygotes. Our previous results indicated that $0.03 \mathrm{mM}$ $\mathrm{H}_{2} \mathrm{O}_{2}$ was the minimum concentration capable of inducing higher intracellular ROS levels and triggering oxidative damage in the zygote $[27,28]$. In this study, we treated zygotes with $0.03 \mathrm{mM} \mathrm{H}_{2} \mathrm{O}_{2}$ at $7 \mathrm{hpi}$ (G1 phase) to induce oxidative stress in zygotes, finding that $\mathrm{H}_{2} \mathrm{O}_{2}$ reduced the rate of blastocyst formation at day 4 but did not reduce the rates of 2-cell formation, 4-cell formation, and 8-cell formation until day 3 as compared with that observed in untreated control zygotes, representing the most similar situation to clinically observed phenomena [29, 30]. Previous studies showed that embryo damage caused by suboptimal culture media during the early stages can reduce the percentage of surviving embryos reaching the blastocyst stage [10]. $\gamma$-H2A histone family member $\mathrm{X}(\gamma \mathrm{H} 2 \mathrm{AX})$ plays an important role in DNA damage response [31] and is often used as a marker for DNA damage [32-34]. Therefore, to determine whether blastocyst reduction results from DNA damage, we identified whether DNA damage occurs in zygotes by immunocytochemical detection of $\gamma \mathrm{H} 2 \mathrm{AX}$ protein.

Our previous study found that zygotes treated with $0.03 \mathrm{mM} \mathrm{H}_{2} \mathrm{O}_{2}$ display a delayed $\mathrm{G} 2 / \mathrm{M}$ phase transition during the first cleavage and that the DNA damage response (DDR) pathway was involved in this delay [28]. However, we previously failed to distinguish whether the delay occurred at G2 phase or M phase in zygotes; therefore, in this study, we performed immunofluorescent identification of $\mathrm{H} 3 \mathrm{~S} 10 \mathrm{P}$, finding that treatment of zygotes with $0.03 \mathrm{mM}$ $\mathrm{H}_{2} \mathrm{O}_{2}$ resulted in prometaphase/metaphase delay. Furthermore, immunofluorescence staining of MAD2L1 and TTK suggested that the SAC is involved in metaphase delay induced by oxidative stress.

\section{Materials and Methods}

2.1. Experimental Animals. Adult Kunming mice (3-6 weeks old) were obtained from the animal center of Shantou University Medical College. Treatment of all mice was in compliance with the Guide for the Care of Use of Laboratory Animals by the National Institutes of Health (NIH publication number 85-23, revised 1996) and the rules set forth by the National Animal Protection of China. All experimental protocols were approved by the Laboratory Animal Ethics Committee of our institution (SUMC2014-014). This study was approved by the Institutional Animal Care and Use Committee of Shantou University Medical College.

2.2. Reagents and Media. Rabbit antiphospho-histone H2AX ( $\gamma$ H2AX; Ser139), rabbit antiphospho-histone H3 (Ser10), and rabbit antiMAD2L1 were purchased from Abcam (Cambridge, UK). Rabbit anti-TTK was obtained from Proteintech (Chicago, IL, USA). Goat antirabbit secondary antibody was obtained from Santa Cruz Biotechnology (Dallas, TX, USA). Human tubal fluid (HTF) was obtained from Sage Science (Beverly, MA, USA). 4,6-diamidino-2-phenylindole (DAPI; $2 \mu \mathrm{g} / \mathrm{mL}$ ) was obtained from Sigma-Aldrich (St. Louis, MO, USA). Antifade fluorescence-mounting medium was purchased from Beyotime Institute of Biotechnology (Haimen, China).

Phosphate-buffered saline (PBS) was dissolved in $1 \mathrm{~L}$ ultrapure water: $8 \mathrm{~g} \mathrm{NaCl}, 0.2 \mathrm{~g} \mathrm{KCl}, 1.44 \mathrm{~g} \mathrm{Na}_{2} \mathrm{HPO}_{4}$, and $0.24 \mathrm{~g} \mathrm{KH}_{2} \mathrm{PO}_{4}$ ( $\mathrm{pH} 7.4$ ). Pancreatin solution was diluted to $0.1 \%$ with $\mathrm{PBS}$, and the $\mathrm{pH}$ adjusted to 3.0 with $\mathrm{HCl}$ prior to and storage at $4^{\circ} \mathrm{C}$. Zygote culture medium was prepared by adding $0.4 \%$ bovine serum albumin (BSA; Sigma-Aldrich) and $10 \%$ fetal bovine serum (FBS; Gibco; Thermo Fisher Scientific, Waltham, MA, USA). The medium for the treated group was created by adding $\mathrm{H}_{2} \mathrm{O}_{2}$ to a final concentration of $0.03 \mathrm{mM}$, followed by preequilibration for $1 \mathrm{~h}$ before use. Fixative liquid consisted of $4 \%$ paraformaldehyde in PBS, and permeabilization buffer consisted of PBS supplemented with $0.5 \%$ Triton X-100. Sperm capacitation liquid (HTF solution supplemented with $1.5 \%$ BSA), fertilization liquid (HTF solution supplemented with $0.4 \%$ BSA), and zygote culture medium were incubated at $37^{\circ} \mathrm{C}$ in a $5 \% \mathrm{CO}_{2}$ incubator and allowed to equilibrate for $4 \mathrm{~h}$ before use. 
2.3. Collection of Sperm and Oocytes, IVF, and Culture of Zygotes. As described in our previous studies [35, 36], male Kunming mice of reproductive age were euthanized, and sperm was collected from the murine cauda epididymis and incubated in capacitation medium (HTF medium containing $1.5 \% \mathrm{BSA}$ ) at $37^{\circ} \mathrm{C}$ in a $5 \% \mathrm{CO}_{2}$ incubator for $1 \mathrm{~h}$. Female mice were induced to superovulate by consecutive intraperitoneal injections of $10 \mathrm{IU}$ pregnant mare serum gonadotropin and $10 \mathrm{IU}$ human chorionic gonadotropin (HCG) $48 \mathrm{~h}$ apart [28], followed by euthanization at $13 \mathrm{~h}$ to $15 \mathrm{~h}$ after HCG administration to obtain cumulus oocytes from the oviducts. Cumulus oocytes were collected in $37^{\circ} \mathrm{C}$ PBS, followed by transfer to prepared $37^{\circ} \mathrm{C}$ fertilization liquid (HTF medium containing $0.4 \% \mathrm{BSA}$ ) under oil and containing $10 \mu \mathrm{L}$ capacitated sperm and incubation at $37^{\circ} \mathrm{C}$ for $6 \mathrm{~h}$ in a $5 \% \mathrm{CO}_{2}$ incubator to permit fertilization. Zygotes were then washed three times and cultured in new medium (HTF medium supplemented with $0.4 \% \mathrm{BSA}$ and $10 \% \mathrm{FBS}$ ).

2.4. Mouse Zygote Model for Oxidative Damage. According to our previous studies, $0.03 \mathrm{mM} \mathrm{H} \mathrm{H}_{2} \mathrm{O}_{2}$ is the minimum concentration capable of inducing oxidative damage in zygotes [27]. Therefore, at $7 \mathrm{hpi}$, we selected two pronuclear zygotes in G1 phase for exposure to culture medium containing $0.03 \mathrm{mM} \mathrm{H}_{2} \mathrm{O}_{2}$ for $30 \mathrm{~min}$ to induce oxidative damage. Zygotes in the control and treated groups were then extensively washed twice in fresh embryo-culture medium, followed by further culture in embryo-culture medium for evaluation.

2.5. Indirect Immunofluorescence Staining for $\gamma H 2 A X$, H3S10P, MAD2L1, and TTK. Zygotes were collected at different times to detect the activation of different proteins $(\gamma \mathrm{H} 2 \mathrm{AX}$ was detected at 17.0-18.0 hpi, $\mathrm{H} 3 \mathrm{~S} 10 \mathrm{P}$ was detected every $30 \mathrm{~min}$ at $18.0-24.0 \mathrm{hpi}$, and MAD2L1 and TTK were detected at 19.0-19.5 hpi and 21.5-22.5 hpi in the control and treated groups, resp.). Zona pellucida was removed from zygotes with $0.1 \%$ pancreatin. After washing for $5 \mathrm{~min}$ three times with PBS containing $0.05 \%$ Tween-20 (TPBS), the zygotes were fixed in $4 \%$ paraformaldehyde in TPBS for $30 \mathrm{~min}$, mounted on polylysine-coated slides, and washed three times again with TPBS. Zygotes were then permeabilized in TPBS supplemented with $0.5 \%$ Triton X-100 for $30 \mathrm{~min}$ at room temperature. After washing three times as above, zygotes were blocked in blocking solution (TPBS supplemented with $10 \%$ normal goat serum and 3\% BSA) for $1 \mathrm{~h}$. After blocking, primary antibodies were incubated at $4^{\circ} \mathrm{C}$ overnight at the following dilutions: rabbit anti- $\gamma \mathrm{H} 2 \mathrm{AX}$ $(1: 300)$, rabbit anti-H3S10P $(1: 150)$, rabbit anti-MAD2L1 $(1: 100)$, and rabbit anti-TTK $(1: 100)$. After washing with TPBS three times, zygotes were incubated with the secondary antibody (goat antirabbit IgG-FITC; $1: 200$ ) at room temperature for $1 \mathrm{~h}$ then washed three times with TPBS. Zygotes were counterstained with DAPI at room temperature for $30 \mathrm{~min}$, washed three times, mounted with antifade fluorescence-mounting medium, and coverslipped. A fluorescence microscope (Nikon Eclipse90 Ni-E; Nikon, Tokyo, Japan) was used to observe the signal.

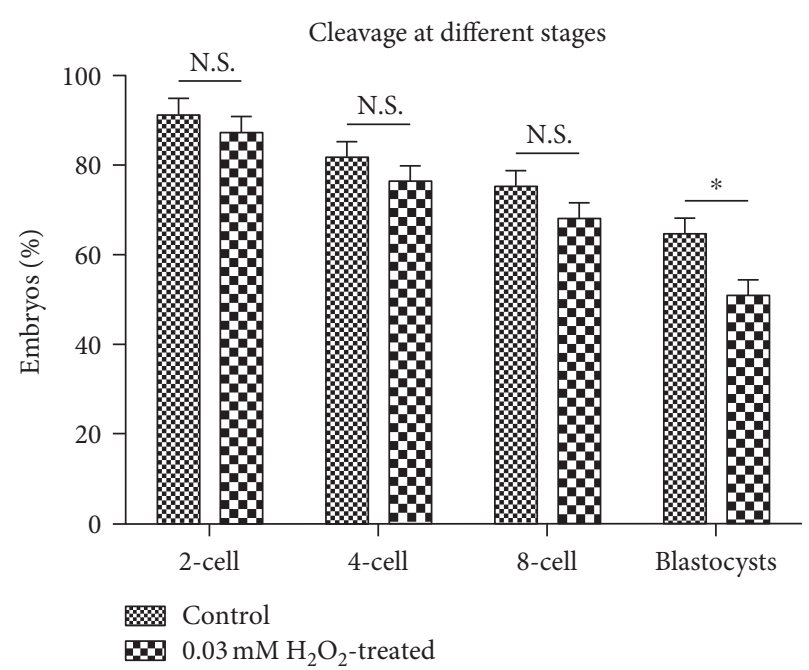

FIgURE 1: Developmental profiles of control and $\mathrm{H}_{2} \mathrm{O}_{2}$-treated embryos. Based on the chi-square test, no statistical differences were observed in the rates of 2-cell formation, 4-cell formation, or 8 -cell formation between the control and treated groups $(P>0.05)$. However, treatment with $0.03 \mathrm{mM} \mathrm{H}_{2} \mathrm{O}_{2}$ produced a significant decrease in the rate of blastocyst formation $(P<0.05) .{ }^{*} P<0.05$.

\section{Results}

3.1. Treatment with $0.03 \mathrm{mM} \mathrm{H}_{2} \mathrm{O}_{2}$ Reduces Blastocyst Formation without Affecting Early Cell Division. To explore the impact of oxidative damage on embryo development in vitro, we monitored cleavage rates at different stages in control and $0.03 \mathrm{mM} \mathrm{H}_{2} \mathrm{O}_{2}$-treated groups. Our results showed that $\mathrm{H}_{2} \mathrm{O}_{2}$ treatment did not significantly reduce the rates of 2-cell formation, 4-cell formation, or 8-cell formation $(P>0.05)$ but produced a significant decrease in the rates of blastocyst formation $(P<0.05)$ (Figure 1$)$, which was similar to phenomena observed in the clinic $[29,30]$. Therefore, we considered that treatment with $0.03 \mathrm{mM}$ $\mathrm{H}_{2} \mathrm{O}_{2}$ reflected clinical relevance and was suitable for inducing oxidative damage in the treated group.

\section{2. $\mathrm{H}_{2} \mathrm{O}_{2}$ Exposure Induces Nuclear $\gamma \mathrm{H} 2 \mathrm{AX}$ Foci Formation} in Mouse Zygotes. $\gamma \mathrm{H} 2 \mathrm{AX}$ is an early and sensitive marker for DNA damage. To determine whether $\mathrm{H}_{2} \mathrm{O}_{2}$ induces DNA damage, we monitored the presence of $\gamma \mathrm{H} 2 \mathrm{AX}$ staining by immunofluorescence microscopy. Punctate $\gamma \mathrm{H} 2 \mathrm{AX}$ staining was observed in the nuclei of $\mathrm{H}_{2} \mathrm{O}_{2}$-treated zygotes but not detected in control zygotes (Figure 2). These findings indicated that $\mathrm{H}_{2} \mathrm{O}_{2}$ treatment resulted in DNA damage.

3.3. The Presence of H3S1OP during Different Phases in Mouse Zygotes. In our study, we observed no H3S10P-positive signals (green) during the $\mathrm{S}$ phase, and subsequent analysis indicated that H3S10P affected only a subset of genes and not the entire chromatin (blue staining with DAPI) in late G2-interphase cells. However, H3S10P prevalence extended along the chromosomes, with completion observed during prometaphase and remaining evident at the metaphase plate, but disappearing during anaphase and telophase (Figure 3). 

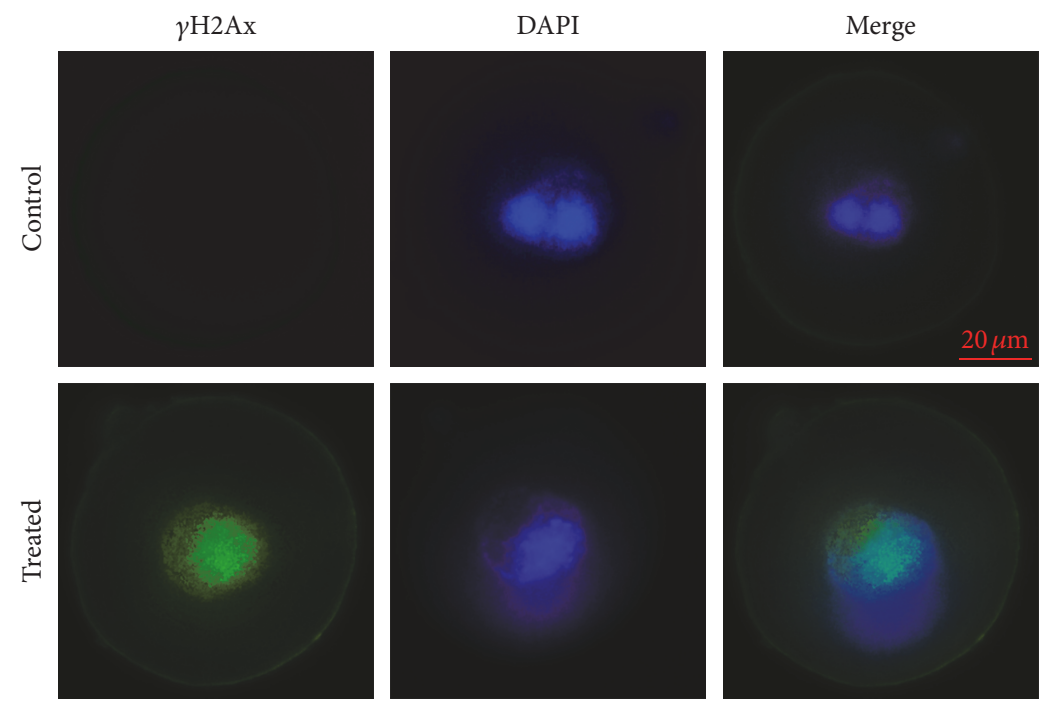

FIGURE 2: $\gamma \mathrm{H} 2 \mathrm{AX}$ detection in mouse zygotes at $17 \mathrm{hpi}$. Nuclei were stained with DAPI (blue). $\gamma \mathrm{H} 2 \mathrm{AX}$ staining was detected in the nuclei of $\mathrm{H}_{2} \mathrm{O}_{2}$-treated embryos, but not in those of the control group.

Therefore, we used H3S10P as a marker of prometaphase/ metaphase during mitosis.

\subsection{Percentage of H3S10P-Positive Zygotes at Different Time} Postfertilization. We monitored the ratios of prometaphase/ metaphase zygotes by immunofluorescence staining for $\mathrm{H} 3 \mathrm{~S} 10 \mathrm{P}$ at $30 \mathrm{~min}$ intervals and $18 \mathrm{hpi}$ to $24 \mathrm{hpi}$ (Table 1 ). After converting the ratios into percentages, we found that the percentage of H3S10P-positive zygotes in the control group was $>50 \%$ at $19.0 \mathrm{hpi}$ and $19.5 \mathrm{hpi}(52.86 \%$ and $55.56 \%$, resp.), reaching a maximum at $19.5 \mathrm{hpi}$ and decreasing to zero at 21.0 hpi (i.e., when zygotes finished the first cleavage). However, in the $\mathrm{H}_{2} \mathrm{O}_{2}$-treated group, the percentage of H3S10P-positive zygotes was $>50 \%$ at $21.5,22.0$, and $22.5 \mathrm{hpi}(51.4 \%, 66.6 \%$, and $65.0 \%$, resp.) and reached a maximum at $22.0 \mathrm{hpi}$, after which $\mathrm{H} 3 \mathrm{~S} 10 \mathrm{P}$ levels decreased rapidly and were no longer observed. These data suggested that prometaphase/metaphase was delayed in $\mathrm{H}_{2} \mathrm{O}_{2}$-treated zygotes and that the duration of prometaphase/metaphase in treated zygotes was prolonged relative to that observed in the control group based on the broader peak width observed in the $\mathrm{H}_{2} \mathrm{O}_{2}$-treated group (Figure 4).

3.5. MAD2L1 and TTK Expression in the $\mathrm{H}_{2} \mathrm{O}_{2}$-Treated and Control Groups. Based on H3S10P prevalence, we chose to examine the expression of MAD2L1 and TTK from $19.0 \mathrm{hpi}$ to $19.5 \mathrm{hpi}$ for the control group and from $21.5 \mathrm{hpi}$ to $22.5 \mathrm{hpi}$ for the treated group. In control zygotes, MAD2L1 expression displayed a punctate pattern throughout the cytoplasm but was enriched in the nucleus in $\mathrm{H}_{2} \mathrm{O}_{2}$-treated zygotes (Figure 5, green). TTK expression was similarly analyzed by indirect immunofluorescence (Figure 6, green), showing positive staining in the $\mathrm{H}_{2} \mathrm{O}_{2}$-treated group in association with the chromatin and no staining detected in the control group. These results suggested that SAC was activated upon oxidative damage.

\section{Discussion}

Cultured embryos are usually subjected to oxidative stress in vitro due to suboptimal culture conditions, and $\mathrm{H}_{2} \mathrm{O}_{2}$ is commonly used in studies investigating the effects of oxidative stress $[2,27,37]$. When DNA damage results from oxidative stress, two pathways are activated in zygotes: the DDR pathway, which monitors DNA integrity, and the SAC, which responds to defects in spindle attachment/ tension in metaphase during mitosis and meiosis [15]. We previously showed that oxidative damage activates the DDR pathway to mediate G2/M cell cycle arrest to allow repair of $\mathrm{H}_{2} \mathrm{O}_{2}$-induced oxidative damage [28]. By contrast, in cycling cells, the SAC is critical for preventing genome instability and producing healthy daughter cells containing the same genetic information as the mother cell during mitosis. In this study, we explored the timing of metaphase during the first round of mitosis in control and $\mathrm{H}_{2} \mathrm{O}_{2}$-treated zygotes and investigated the involvement of the SAC in metaphase delay.

$\gamma \mathrm{H} 2 \mathrm{AX}$ is an early indicator of DNA damage [31], and we previously suggested that $\gamma \mathrm{H} 2 \mathrm{AX}$ plays an important role in both DDR [28] and SAC by contributing to the recruitment of both TTK and MAD2L1 to the kinetochore during metaphase [38]. Our results in this study (Figure 2) showed that oxidative stress-induced DNA damage existed in $\mathrm{H}_{2} \mathrm{O}_{2}$-treated zygotes, but not in control zygotes. Additionally, we revealed that $\mathrm{H}_{2} \mathrm{O}_{2}$ treatment of zygotes did not reduce the rates of 2-cell formation, 4-cell formation, or 8cell formation but did reduce the rates of blastocyst formation at day 4 . This indicated that a moderate concentration of $\mathrm{H}_{2} \mathrm{O}_{2}(0.03 \mathrm{mM})$ produced a similar outcome as that observed clinically and in previous studies [27, 29, 30]. Therefore, this $\mathrm{H}_{2} \mathrm{O}_{2}$ concentration was used to treat embryos in our study.

H3S10P is a marker for prometaphase/metaphase [39] and can be used to identify metaphase delay upon DNA 

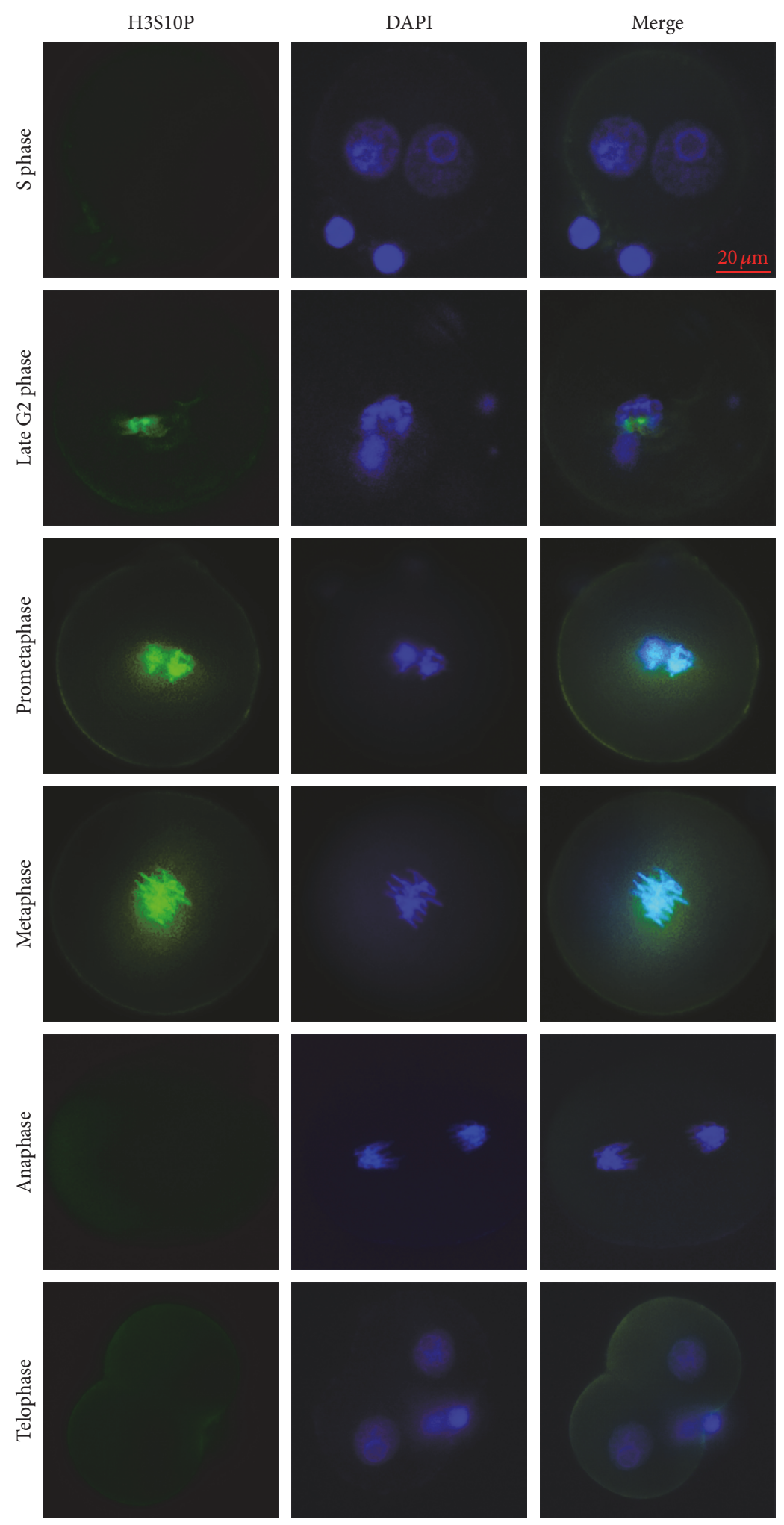

FIGURE 3: H3S10P detection in mouse zygotes. H3S10P (green) expression during $\mathrm{S}$ phase, prometaphase, and anaphase in control zygotes at 18.0, 19.0, and $20.5 \mathrm{hpi}$, respectively. The late G2 phase, metaphase, and telophase visualizations were obtained from treated zygotes at 21.0 , 22.0, and $23.5 \mathrm{hpi}$, respectively. Nuclei were stained with DAPI (blue).

damage in zygotes [15]. Here, we showed that the peak percentage of H3S10P-positive cells in untreated embryos occurred at $19.5 \mathrm{hpi}$ and that after $21 \mathrm{hpi}, \mathrm{H} 3 \mathrm{~S} 10 \mathrm{P}$ was no longer detected as a result of all control zygotes having finished their cleavage. However, in the $\mathrm{H}_{2} \mathrm{O}_{2}$-treated group, the percentage of $\mathrm{H} 3 \mathrm{~S} 10 \mathrm{P}$-positive cells peaked at 
TABLE 1: Ratio of H3S10P-expressing cells in control and treated zygotes from 18 hpi to $21 \mathrm{hpi}$.

\begin{tabular}{lccccccccccccc}
\hline Groups & $\begin{array}{c}18.0 \\
\text { (hpi) }\end{array}$ & $\begin{array}{c}18.5 \\
(\mathrm{hpi})\end{array}$ & $\begin{array}{c}19.0 \\
(\mathrm{hpi})\end{array}$ & $\begin{array}{c}19.5 \\
(\mathrm{hpi})\end{array}$ & $\begin{array}{c}20.0 \\
(\mathrm{hpi})\end{array}$ & $\begin{array}{c}20.5 \\
(\mathrm{hpi})\end{array}$ & $\begin{array}{c}21.0 \\
(\mathrm{hpi})\end{array}$ & $\begin{array}{c}21.5 \\
\text { (hpi) }\end{array}$ & $\begin{array}{c}22.0 \\
\text { (hpi) }\end{array}$ & $\begin{array}{c}22.5 \\
(\mathrm{hpi})\end{array}$ & $\begin{array}{c}23.0 \\
(\mathrm{hpi})\end{array}$ & $\begin{array}{c}23.5 \\
(\mathrm{hpi})\end{array}$ & $\begin{array}{c}24.0 \\
(\mathrm{hpi})\end{array}$ \\
\hline Control & $0 / 67$ & $10 / 56$ & $37 / 70$ & $40 / 72$ & $21 / 75$ & $12 / 70$ & $0 / 63$ & 0 & 0 & 0 & 0 & 0 & 0 \\
Treated & $0 / 52$ & $0 / 53$ & $7 / 52$ & $20 / 86$ & $0 / 49$ & $10 / 70$ & $12 / 60$ & $36 / 70$ & $46 / 69$ & $39 / 60$ & $16 / 64$ & $4 / 58$ & 0 \\
\hline
\end{tabular}

The treated group was administered $0.03 \mathrm{mM} \mathrm{H}_{2} \mathrm{O}_{2}$. Hpi: hours postinsemination.

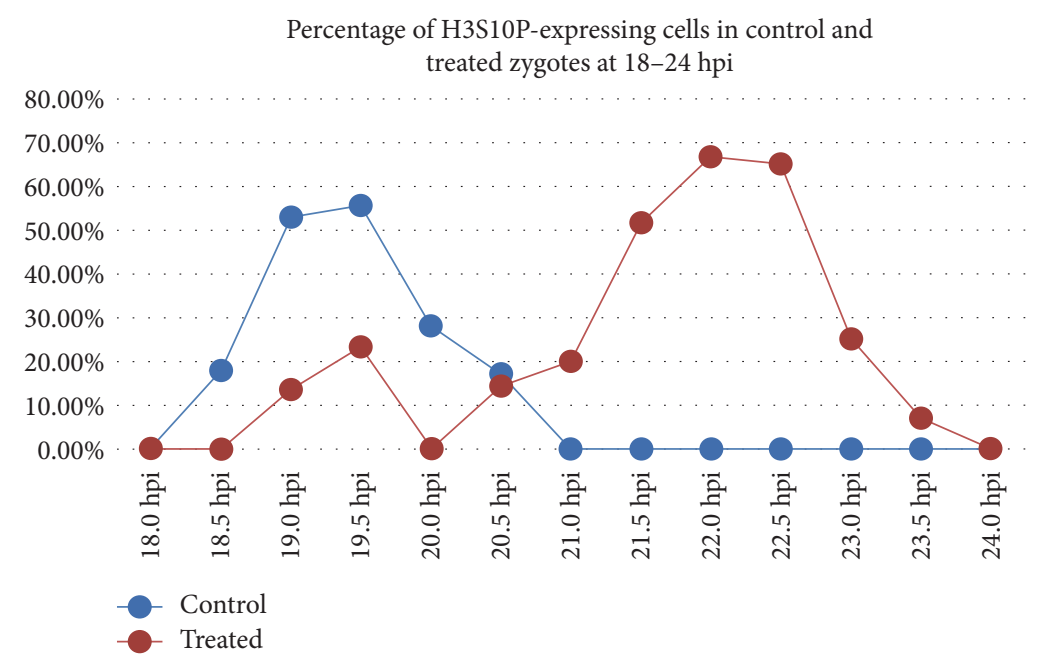

Figure 4: Percentage of cells showing H3S10P prevalence in control and treated zygotes from 18 hpi to 24 hpi. The peak of H3S10P prevalence occurred later in treated zygotes.
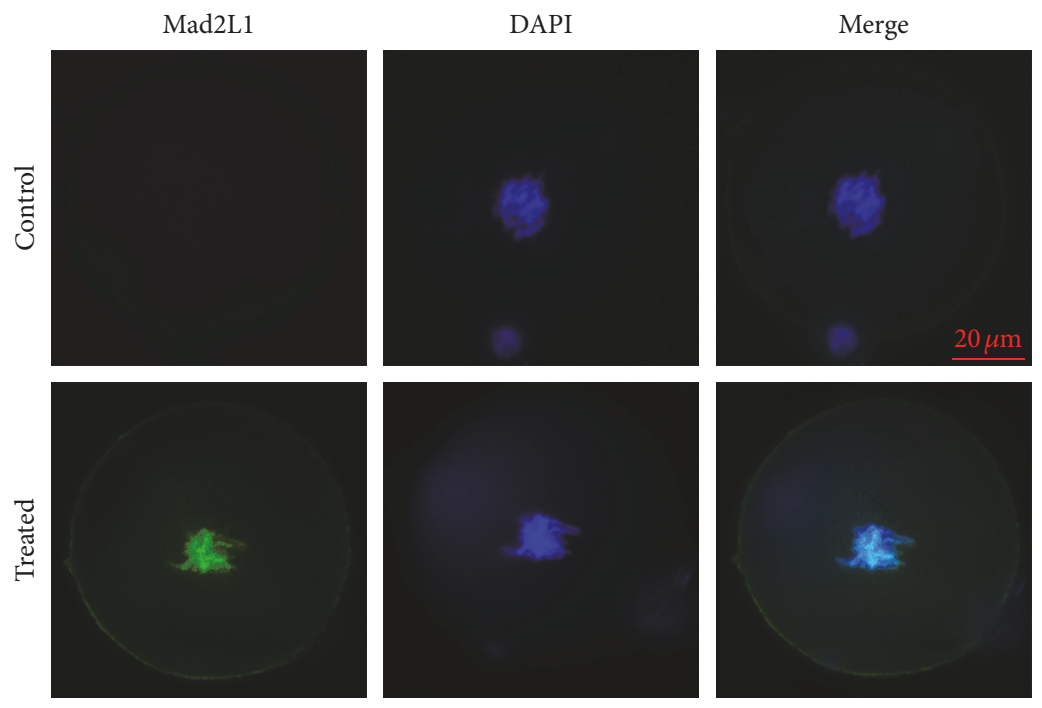

FIGURE 5: MAD2L1 expression is induced in mouse zygotes following treatment with $0.03 \mathrm{mM} \mathrm{H}_{2} \mathrm{O}_{2}$. MAD2L1 immunofluorescence (green) was undetected in untreated cells but detected following treatment of zygotes with $\mathrm{H}_{2} \mathrm{O}_{2}$. Nuclei (blue) were stained with DAPI.

22.0 hpi, indicating that prometaphase/metaphase entry was delayed. Additionally, the duration of prometaphase/ metaphase in treated zygotes was prolonged relative to that observed in the control group. Because the SAC can delay chromosome segregation at prometaphase or metaphase in response to DNA damage $[14,15]$, we considered the delay observed in the treated group as resulting from SAC activation. Furthermore, we observed a small peak at $19.5 \mathrm{hpi}$ in the treated group prior to the appearance of the maximum peak and suggesting zygote heterogeneity in response to oxidative damage, with some zygotes either being insensitive to mild oxidative damage or exhibiting an inactive checkpoint. Based on these results, we chose times from $19.0 \mathrm{hpi}$ to $19.5 \mathrm{hpi}$ and $21.5 \mathrm{hpi}$ to $22.5 \mathrm{hpi}$ for detection of the SAC markers MAD2LI and TTK in control and treated zygotes, respectively. 

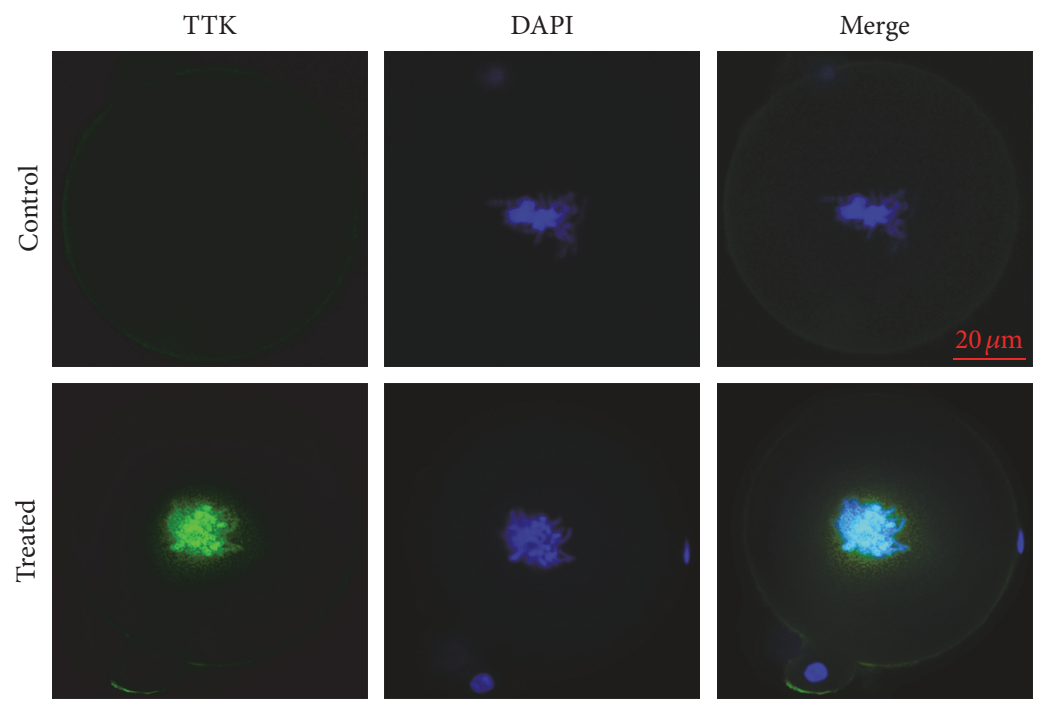

FIGURE 6: TTK expression in mouse zygotes is induced following exposure to $0.03 \mathrm{mM} \mathrm{H}_{2} \mathrm{O}_{2}$. TTK immunofluorescence (green) was undetected in untreated cells but detected following treatment of zygotes with $\mathrm{H}_{2} \mathrm{O}_{2}$. Nuclei (blue) were stained with DAPI.

The metaphase-anaphase transition is mediated by the ubiquitin protein ligase APC/C [40] under the control of a network of regulatory factors, including cell division cycle protein 20 (CDC20), cadherin 1 (CDH1), and MAD2L1. $\mathrm{CDC} 20$ and $\mathrm{CDH} 1$ can activate APC, whereas MAD2L1 acts as an APC/C inhibitor. When the SAC is activated, MAD2L1 forms a ternary complex with CDC20 and the protein kinase BUBR1 to form the mitotic checkpoint complex (MCC) and prevent $\mathrm{APC} / \mathrm{C}$ activation, thereby arresting cells at prometaphase [41]. As a critical mediator of MCC formation and APC/C activation [26, 42], MAD2L1 also plays a key role in SAC functions [19]; therefore, its detection represents a significant determinant of SAC activated. Here, our results (Figure 5) showed that MAD2L1 was enriched in the nucleus in response to oxidative damage, providing further evidence of SAC activation following oxidative damage in zygotes. The SAC monitors microtubule tension and attachment at kinetochores to ensure proper chromosome segregation [43], and MAD2L1 localization in the nuclei is regulated by attachment, not tension. Even a single unattached kinetochore is sufficient to activate MAD2L1 nucleocytoplasmic transport and delay anaphase [20]. Therefore, our findings suggested that oxidative stress was capable of activating the SAC based on dysregulated kinetochoremicrotubule attachment.

In addition to the spatial and temporal regulation of MAD2L1 localization during cell cycle progression and SAC activation, two MAD2L1 conformations with distinct topologies are also vital to the SAC: the open $(\mathrm{O})$ and closed (C) MAD2L1 conformation [26]. Only the C-MAD2L1 conformation obtained following associating with MAD1 or CDC20 can allow blockage of anaphase progression by inhibiting APC/C activation for SAC signaling [22, 26, 42]. A significant function associated with TTK involving cell cycle control concerns conversion of cytosolic MAD2L1 from an inactive open conformation (O-MAD2L1) to a closed conformation (C-MAD2L1) [21]. Another important finding of this study was elevated TTK expression in zygotes exposed to oxidative stress, suggesting a relationship between TTK expression and oxidative stress. Our findings also suggested that during SAC signaling, MAD2L1 recruitment to kinetochores by TTK also occurred upon the initiation of oxidative damage in zygotes [21, 22, 27]. However, TTK only appears to be required for SAC function following spindle damage and not during unperturbed mitosis [44, 45]. By contrast, other SAC components restrain mitosis in the absence of spindle damage [45]. Therefore, as an indicator of SAC activation, our observation of TTK expression in $\mathrm{H}_{2} \mathrm{O}_{2}$-treated zygotes represented additional evidence of SAC activation following oxidative damage.

The SAC is required for regulating mitotic cell cycle progression to ensure mitotic fidelity during preimplantation development in mouse cleavage-stage embryos $[9,46]$. The primary role of the SAC is to ensure that all chromosomes are bioriented and accurately attached to spindle microtubules prior to anaphase initiation, thereby contributing to the prevention of aneuploidy by blocking anaphase onset until correct kinetochore-microtubule attachment and tension are attained [16]. Otherwise, errors in chromosome segregation or distribution might result in aneuploidembryo formation, potentially resulting in implantation failure, spontaneous abortion, genetic diseases, or embryonic death [9]. Embryonic aneuploidy in gametes or early embryos is already the leading cause of IVF failure [7, 9]. Studies of the relationships between oxidative stress and SAC aid in promoting strategies to avoid spontaneous abortion and low rates of reproductive success. However, a more detailed understanding of the mechanisms associated with SAC activation and aneuploid-embryo formation related to oxidative stress is necessary.

\section{Conclusion}

Our results showed that treatment of zygotes with $0.03 \mathrm{mM}$ $\mathrm{H}_{2} \mathrm{O}_{2}$ did not influence the formation of 2-cell embryos, 4-cell embryos, or 8-cell embryos but did reduce rates of 
blastocyst formation, which was similar to developmental phenomena observed clinically in relation to embryos experiencing oxidative damage. Additionally, we observed DNA damage $\mathrm{H}_{2} \mathrm{O}_{2}$-treated zygotes based on the detection of $\gamma \mathrm{H} 2 \mathrm{AX}$, the induction of metaphase delay during initial mitosis of zygotes, and the time of appearance of H3S10P. Furthermore, detection of MAD2L1 and TTK expression by immunofluorescence suggested the involvement of SAC activation in this delay. Given that SAC is largely responsible for chromosome stability, its activation is conducive to avoiding spontaneous abortion resulting from aneuploidy.

\section{Conflicts of Interest}

The authors declare that there is no conflict of interests regarding the publication of this paper.

\section{Acknowledgments}

This study was supported by the National Natural Science Foundation of China (no. 30872771, no. 81070542, no. 81471522, and no. 81671536), the Natural Science Foundation of Guangdong Province of China (no. 8151503102000010, no. 10151503102000020, and no. 2014A030313482), and the Guangdong Provincial Science and Technology Project (2016A020218015).

\section{References}

[1] M. Takahashi, "Oxidative stress and redox regulation on in vitro development of mammalian embryos," Journal of Reproduction and Development, vol. 58, no. 1, pp. 1-9, 2012.

[2] N. T. Bain, P. Madan, and D. H. Betts, "The early embryo response to intracellular reactive oxygen species is developmentally regulated," Reproduction Fertility and Development, vol. 23, no. 4, pp. 561-575, 2011.

[3] N. Kimura, S. Tsunoda, Y. Iuchi, H. Abe, K. Totsukawa, and J. Fujii, "Intrinsic oxidative stress causes either 2-cell arrest or cell death depending on developmental stage of the embryos from SOD1-deficient mice," Molecular Human Reproduction, vol. 16, no. 7, pp. 441-451, 2010.

[4] L. Liu and D. L. Keefe, "Cytoplasm mediates both development and oxidation-induced apoptotic cell death in mouse zygotes," Biology of Reproduction, vol. 62, no. 6, pp. 1828-1834, 2000.

[5] J. J. Gille and H. Joenje, "Cell culture models for oxidative stress: superoxide and hydrogen peroxide versus normobaric hyperoxia," Mutation Research/DNAging, vol. 275, no. 3-6, pp. 405-414, 1992.

[6] L. L. Fava, M. Kaulich, E. A. Nigg, and A. Santamaria, "Probing the in vivo function of Mad1:C-Mad2 in the spindle assembly checkpoint," The EMBO Journal, vol. 30, no. 16, pp. 33223336, 2011.

[7] H. Bolton, S. J. Graham, N. Van der Aa et al., "Mouse model of chromosome mosaicism reveals lineage-specific depletion of aneuploid cells and normal developmental potential," Nature Communications, vol. 7, article 11165, 2016.

[8] W. H. Wang and Q. Y. Sun, "Meiotic spindle, spindle checkpoint and embryonic aneuploidy," Frontiers in Bioscience, vol. 11, pp. 620-636, 2006.

[9] Y. Wei, S. Multi, C. R. Yang et al., "Spindle assembly checkpoint regulates mitotic cell cycle progression during preimplantation embryo development," PLoS One, vol. 6, no. 6, article e21557, 2011.

[10] P. Ventura-Juncá, I. Irarrázaval, A. J. Rolle, J. I. Gutiérrez, R. D. Moreno, and M. J. Santos, "In vitro fertilization (IVF) in mammals: epigenetic and developmental alterations. Scientific and bioethical implications for IVF in humans," Biological Research, vol. 48, p. 68, 2015.

[11] M. R. Thomas, A. Sparks, G. Ryan, and B. J. Van Voorhis, "Clinical predictors of human blastocyst formation and pregnancy after extended embryo culture and transfer," Fertility and Sterility, vol. 94, no. 2, pp. 543-548, 2010.

[12] A. Meuter, L.-M. Rogmann, B. J. Winterhoff, T. Tchkonia, J. L. Kirkland, and D. E. Morbeck, "Markers of cellular senescence are elevated in murine blastocysts cultured in vitro: molecular consequences of culture in atmospheric oxygen," Journal of Assisted Reproduction and Genetics, vol. 31, no. 10, pp. 12591267, 2014.

[13] A. Musacchio and E. D. Salmon, "The spindle-assembly checkpoint in space and time," Nature Reviews Molecular Cell Biology, vol. 8, no. 5, pp. 379-393, 2007.

[14] E. A. Foley and T. M. Kapoor, "Microtubule attachment and spindle assembly checkpoint signalling at the kinetochore," Nature Reviews Molecular Cell Biology, vol. 14, no. 1, pp. 2537, 2013.

[15] K. S. Lawrence, T. Chau, and J. Engebrecht, "DNA damage response and spindle assembly checkpoint function throughout the cell cycle to ensure genomic integrity," PLoS Genetics, vol. 11, no. 4, article e1005150, 2015.

[16] Z. Maciejewska, Z. Polanski, K. Kisiel, J. Z. Kubiak, and M. A. Ciemerych, "Spindle assembly checkpoint-related failure perturbs early embryonic divisions and reduces reproductive performance of LT/Sv mice," Reproduction, vol. 137, no. 6, pp. 931-942, 2009.

[17] M. J. Hendzel, Y. Wei, M. A. Mancini et al., "Mitosisspecific phosphorylation of histone $\mathrm{H} 3$ initiates primarily within pericentromeric heterochromatin during G2 and spreads in an ordered fashion coincident with mitotic chromosome condensation," Chromosoma, vol. 106, no. 6, pp. 348360, 1997.

[18] B. Perez-Cadahia, B. Drobic, and J. R. Davie, "H3 phosphorylation: dual role in mitosis and interphase," Biochemistry and Cell Biology, vol. 87, no. 5, pp. 695-709, 2009.

[19] M. Sikora-Polaczek, A. Hupalowska, Z. Polanski, J. Z. Kubiak, and M. A. Ciemerych, "The first mitosis of the mouse embryo is prolonged by transitional metaphase arrest," Biology of Reproduction, vol. 74, no. 4, pp. 734743, 2006.

[20] J. C. Waters, R. Chen, A. W. Murray, and E. D. Salmon, "Localization of Mad2 to kinetochores depends on microtubule attachment, not tension," The Journal of Cell Biology, vol. 141, no. 5, pp. 1181-1191, 1998.

[21] L. Hewitt, A. Tighe, S. Santaguida et al., "Sustained Mps1 activity is required in mitosis to recruit O-Mad2 to the Mad1-CMad2 core complex," The Journal of Cell Biology, vol. 190, no. 1, pp. 25-34, 2010.

[22] M. Maldonado and T. M. Kapoor, "Constitutive Mad1 targeting to kinetochores uncouples checkpoint signalling from chromosome biorientation," Nature Cell Biology, vol. 13, no. 4, pp. 475-482, 2011.

[23] X. Luo, Z. Tang, J. Rizo, and H. Yu, "The Mad2 spindle checkpoint protein undergoes similar major conformational 
changes upon binding to either Mad1 or Cdc20," Molecular Cell, vol. 9, no. 1, pp. 59-71, 2002.

[24] L. Sironi, M. Mapelli, S. Knapp, A. De Antoni, K. T. Jeang, and A. Musacchio, "Crystal structure of the tetrameric Mad1Mad2 core complex: implications of a 'safety belt' binding mechanism for the spindle checkpoint," The EMBO Journal, vol. 21, no. 10, pp. 2496-2506, 2002.

[25] A. DeAntoni, V. Sala, and A. Musacchio, "Explaining the oligomerization properties of the spindle assembly checkpoint protein Mad2," Philosophical Transactions of the Royal Society of London: Series B, Biological Sciences, vol. 360, no. 1455, pp. 637-647, 2005.

[26] M. Mapelli, L. Massimiliano, S. Santaguida, and A. Musacchio, "The Mad2 conformational dimer: structure and implications for the spindle assembly checkpoint," Cell, vol. 131, no. 4, pp. 730-743, 2007.

[27] D. Qian, Z. Li, Y. Zhang et al., "Response of mouse zygotes treated with mild hydrogen peroxide as a model to reveal novel mechanisms of oxidative stress-induced injury in early embryos," Oxidative Medicine and Cellular Longevity, vol. 2016, Article ID 1521428, 11 pages, 2016.

[28] Y. Zhang, D. Qian, Z. Li et al., "Oxidative stress-induced DNA damage of mouse zygotes triggers G2/M checkpoint and phosphorylates Cdc25 and Cdc2," Cell Stress \& Chaperones, vol. 21, no. 4, pp. 687-696, 2016.

[29] H. N. Ciray, T. Aksoy, K. Yaramanci, I. Karayaka, and M. Bahceci, "In vitro culture under physiologic oxygen concentration improves blastocyst yield and quality: a prospective randomized survey on sibling oocytes," Fertility and Sterility, vol. 91, 4 Supplement, pp. 1459-1461, 2009.

[30] E. Kasterstein, D. Strassburger, D. Komarovsky et al., "The effect of two distinct levels of oxygen concentration on embryo development in a sibling oocyte study," Journal of Assisted Reproduction and Genetics, vol. 30, no. 8, pp. 1073-1079, 2013.

[31] S. Benzina, A. Pitaval, C. Lemercier et al., "A kinome-targeted RNAi-based screen links FGF signaling to H2AX phosphorylation in response to radiation," Cellular and Molecular Life Sciences, vol. 72, no. 18, pp. 3559-3573, 2015.

[32] V. Turinetto and C. Giachino, "Multiple facets of histone variant H2AX: a DNA double-strand-break marker with several biological functions," Nucleic Acids Research, vol. 43, no. 5, pp. 2489-2498, 2015.

[33] C. E. Redon, A. J. Nakamura, Y. Zhang et al., "Histone $\gamma \mathrm{H} 2 \mathrm{AX}$ and poly(ADP ribose) as clinical pharmacodynamic biomarkers," Clinical Cancer Research, vol. 16, no. 18, pp. 45324542, 2010.

[34] A. Li, Y. Yu, S.-C. Lee, T. Ishibashi, S. P. Lees-Miller, and J. Ausió, "Phosphorylation of histone H2A.X by DNAdependent protein kinase is not affected by core histone acetylation, but it alters nucleosome stability and histone H1 binding," The Journal of Biological Chemistry, vol. 285, no. 23, pp. 17778-17788, 2010.

[35] Y. Song, Z. Li, B. Wang, J. Xiao, X. Wang, and J. Huang, "Phospho-Cdc25 correlates with activating G2/M checkpoint in mouse zygotes fertilized with hydrogen peroxide-treated mouse sperm," Molecular and Cellular Biochemistry, vol. 396, no. 1-2, pp. 41-48, 2014.

[36] B. Wang, Z. Li, C. Wang et al., "Zygotic G2/M cell cycle arrest induced by ATM/Chk1 activation and DNA repair in mouse embryos fertilized with hydrogen peroxide-treated epididymal mouse sperm," PLoS One, vol. 8, no. 9, article e73987, 2013.
[37] L. Liu, J. R. Trimarchi, and D. L. Keefe, "Involvement of mitochondria in oxidative stress-induced cell death in mouse zygotes," Biology of Reproduction, vol. 62, no. 6, pp. 1745-1753, 2000.

[38] F. Dotiwala, J. C. Harrison, S. Jain, N. Sugawara, and J. E. Haber, "Mad2 prolongs DNA damage checkpoint arrest caused by a double-strand break via a centromere-dependent mechanism," Current Biology, vol. 20, no. 4, pp. 328-332, 2010.

[39] C. Prigent and S. Dimitrov, "Phosphorylation of serine 10 in histone H3, what for?," Journal of Cell Science, vol. 116, Part 18, pp. 3677-3685, 2003.

[40] K. Nasmyth, "How do so few control so many?," Cell, vol. 120, no. 6, pp. 739-746, 2005.

[41] G. Fang, H. Yu, and M. W. Kirschner, "Control of mitotic transitions by the anaphase-promoting complex," Philosophical Transactions of the Royal Society of London: Series B, Biological Sciences, vol. 354, no. 1389, pp. 1583-1590, 1999.

[42] W. C. Chao, K. Kulkarni, Z. Zhang, E. H. Kong, and D. Barford, "Structure of the mitotic checkpoint complex," Nature, vol. 484, no. 7393, pp. 208-213, 2012.

[43] K. B. Shannon, J. C. Canman, and E. D. Salmon, "Mad2 and BubR1 function in a single checkpoint pathway that responds to a loss of tension," Molecular Biology of the Cell, vol. 13, no. 10, pp. 3706-3719, 2002.

[44] V. M. Stucke, C. Baumann, and E. A. Nigg, "Kinetochore localization and microtubule interaction of the human spindle checkpoint kinase Mps1," Chromosoma, vol. 113, no. 1, pp. 1-15, 2004.

[45] M. Schmidt, Y. Budirahardja, R. Klompmaker, and R. H. Medema, "Ablation of the spindle assembly checkpoint by a compound targeting Mps1," EMBO Reports, vol. 6, no. 9, pp. 866-872, 2005.

[46] P. Meraldi, V. M. Draviam, and P. K. Sorger, "Timing and checkpoints in the regulation of mitotic progression," Developmental Cell, vol. 7, no. 1, pp. 45-60, 2004. 


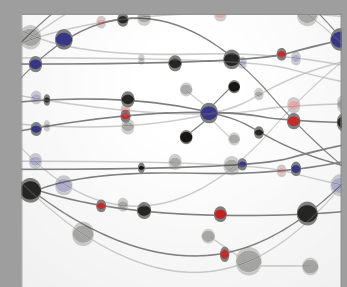

The Scientific World Journal
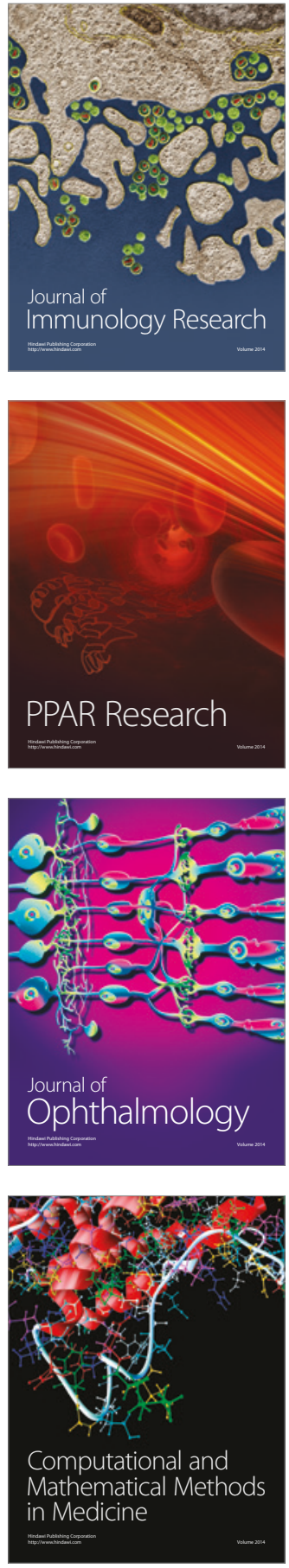

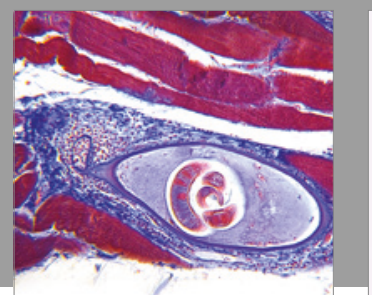

Gastroenterology Research and Practice
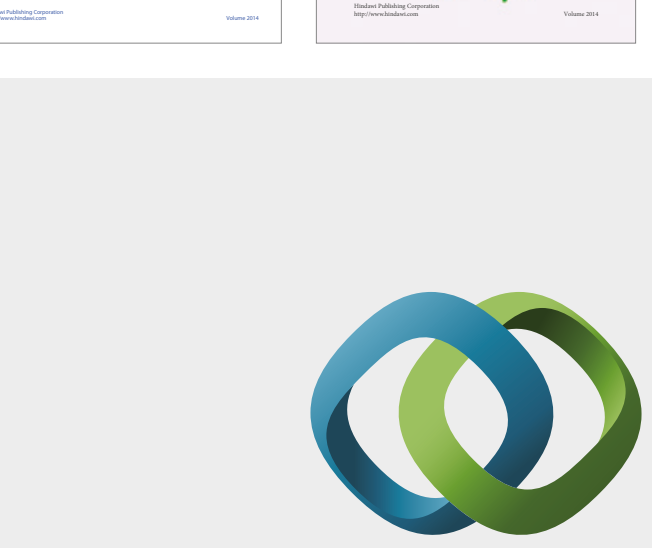

\section{Hindawi}

Submit your manuscripts at

https://www.hindawi.com
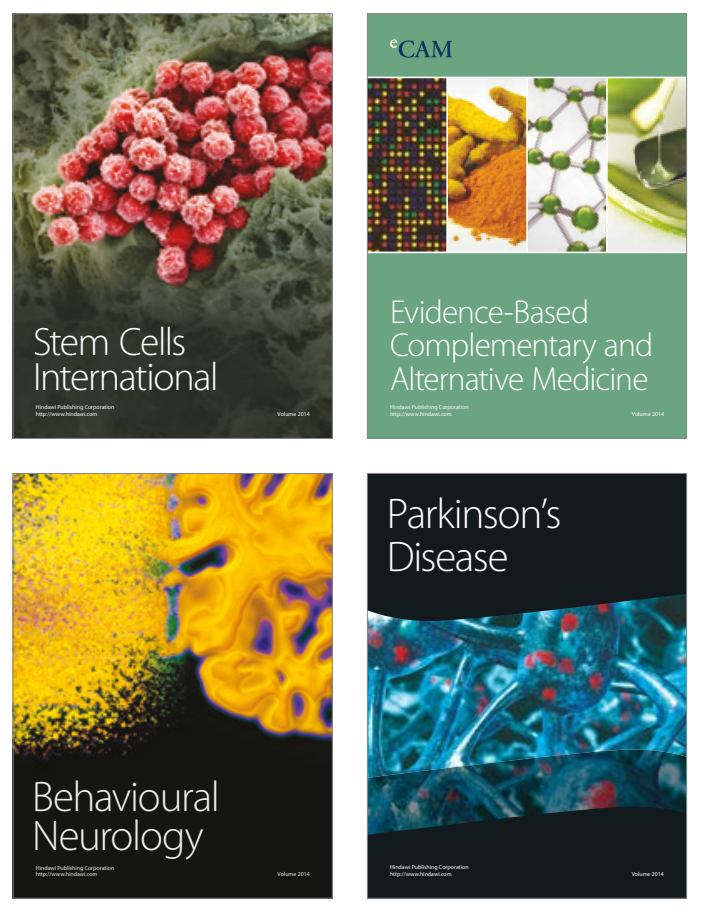
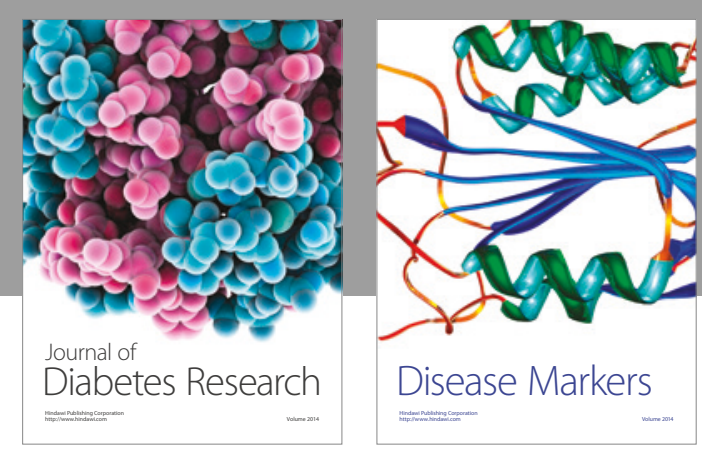

Disease Markers
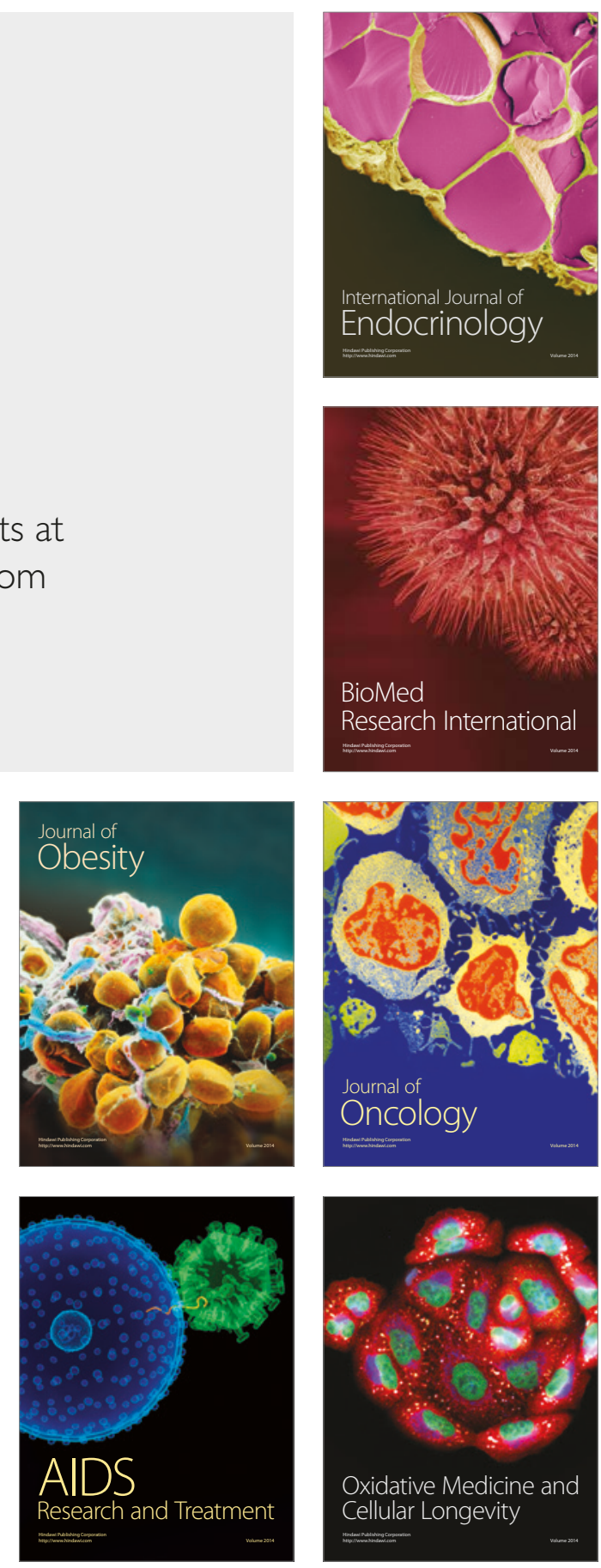